\title{
Specialized concussion clinic referrals are not intended for all acute concussion patients in the emergency department
}

\author{
Catherine E. Varner, MD, MSc ${ }^{*} \ddagger$
}

The emergency department (ED) is the first point of healthcare contact for most head injured patients. The majority (estimated $85 \%$ ) of patients suffering a head injury will not have intra-cranial damage requiring neurosurgical consultation but may have symptoms of mild traumatic brain injury, also known as concussion. ${ }^{1}$ Early and spontaneous resolution occurs in most patients within 7 days, yet $15 \%-30 \%$ of patients with concussion will develop post-concussion syndrome. Patients with post-concussion syndrome have persistent symptoms that usually last 2-4 months and typically peak 4-6 weeks following the injury. However, in a smaller percentage of patients, symptoms last for a year or longer. Approximately $20 \%$ of adults with post-concussion syndrome will not have returned to full-time work 1 year after the initial injury, and some are disabled permanently. ${ }^{2}$ To date, clinical prediction tools do not yet exist to accurately identify adult concussion patients at risk of post-concussion syndrome.

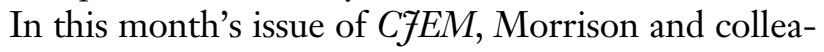
gues performed a retrospective chart review of adult patients diagnosed with concussion in 2016 at the Hamilton Health Sciences ED and Urgent Care Centre. ${ }^{3}$ Of the 389 included patients, 38 (9.8\%) returned to the ED within 14 days. Of patients returning to the ED, 25 (65.8\%) returned with a chief complaint of headache, and $16(42.1 \%)$ underwent head CTs at a subsequent ED visit, without any abnormal findings on radiologist interpretation. The authors also report patients who suffered a sport-related concussion or those referred to a specialized clinic were less likely to return, and patients with assault-related concussion were more likely to return to the ED.

At first glance, these findings are in keeping with other concussion studies of adult ED populations, and the characteristics of this cohort are generalizable to most Canadian EDs. However, in the interpretation of the results, the authors do not acknowledge the influence of unmeasured confounding variables associated with re-seeking unscheduled acute care. ${ }^{3}$ One example is attributing assault victims' higher rates of return ED visits to document ongoing symptoms for the purposes of legal action. Patients with concussion resulting from assault or other violence have a higher risk of posttraumatic stress disorder and ongoing psychological symptoms for prolonged periods following injury. ${ }^{4} \mathrm{Vic}-$ tims of assault are more likely to have pre-existing psychiatric and medical comorbidities that influence care-seeking behaviors, all unaccounted for in this cohort. $^{5}$

The authors also conclude that referral to a specialized concussion clinic may mitigate return visits to the ED. While there is little evidence to dispute that return ED visits may decrease with an urgent referral to specialized clinic, studies have not evaluated whether a referral to a specialized concussion clinic immediately following injury results in improved patient outcomes, including prevention of post-concussion syndrome or return to pre-injury activities. In 2018, the Ontario Neurotrauma Foundation released Standards for Post-Concussion

From the *Schwartz/Reisman Emergency Medicine Institute; †Department of Family and Community Medicine; and the $¥$ University of Toronto,Sinai Health System, Toronto, ON.

Correspondence to: Dr. Catherine Varner, Schwartz/Reisman Emergency Medicine Institute, Sinai Health System, Department of Family and Community Medicine, University of Toronto, 206-600 University Avenue, Toronto, ON M5 G 1X5; Email: catherine.varner@ sinaihealthsystem.ca

(C) Canadian Association of Emergency Physicians

CJEM 2019;21(6):694-697

DOI 10.1017/cem.2019.430 


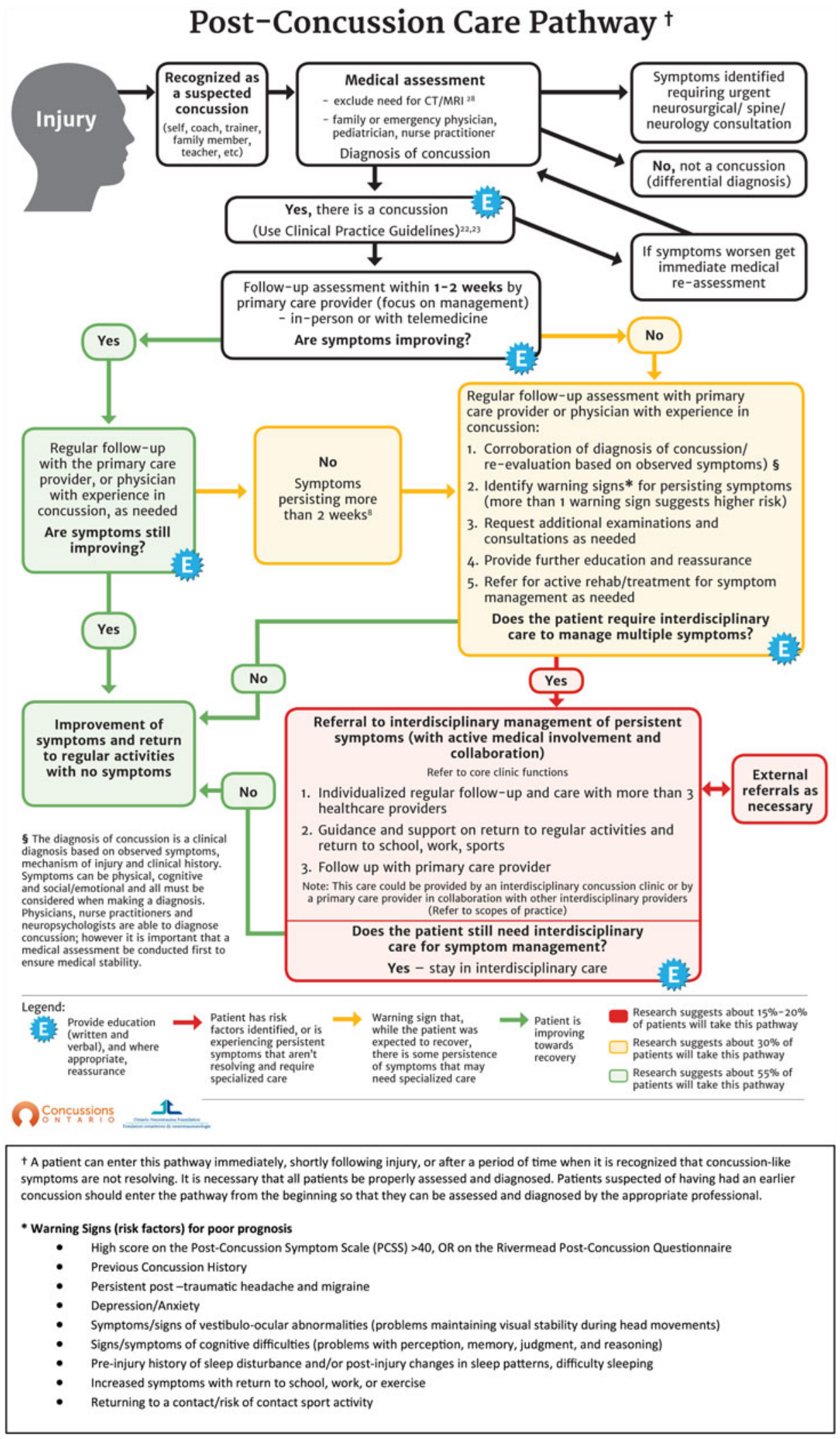

Figure 1. Post-concussion care pathway from the Ontario Neurotrauma Foundation Standards of Post-Concussion Care. 
Care that define standards for specialized concussion clinics and also outline their role in post-concussion care. ${ }^{6}$ The standards emphasize that the majority of patients will have resolution of symptoms within 7 days, and all patients should have follow-up with their primary care providers within 1 to 2 weeks following injury. Referral to a specialized clinic is only recommended if symptoms are persistent 4 weeks after injury or earlier if risk factors for developing post-concussion syndrome are present (Figure 1). ${ }^{6}$ Heeding these standards may ensure that patients with persistent symptoms or those who are at high risk of post-concussion syndrome can access these clinics, because high quality, specialized concussion care is limited in Canada.

A 2017 systematic review of early educational interventions in the $E D$ for concussion concluded that there are no clear and consistent interventions or standard management practices that exist for acute concussion patients in the ED. ${ }^{7}$ However, emerging data suggest that early exercise interventions may have favorable effects on concussion recovery. ${ }^{8,9}$ In a $20167 A M A$ article, Grool et al. examined the association between return to early physical activity and symptom resolution, and the findings were both surprising and contradictory to existing treatment recommendations. ${ }^{8}$ This prospective, multicentre cohort study followed 3,063 concussed adolescents and children and found that an early return to physical activity within 1 week after injury was associated with hastened symptom recovery. Among participants ages 5 to 18 years with acute concussion, physical activity within 7 days of acute injury compared with no physical activity was associated with reduced postconcussive symptoms by an absolute risk reduction of $11.4 \%$ (95\% CI: $5.8 \%$ to $16.9 \%$ ) at 28 days. In a 2019 article in 7 AMA Pediatrics by Leddy et al., acute, adolescent concussion patients were randomized to either early subthreshold aerobic exercise 2 days post-injury or a placebo-like stretching program. ${ }^{9}$ Aerobic exercise participants recovered in a median of 4 days sooner than the stretching group and had a nonsignificant lower incidence of delayed recovery.

As demonstrated by Morrison et al., adults presenting to the ED with concussion have differing demographic and injury-characteristics than previously studied pediatric populations, particularly with respect to mechanism of injury. ${ }^{3,8}$ It is uncertain whether early exercise interventions will have the same impact on adult recovery as they have in children and adolescents. Results from a randomized controlled trial comparing prescribed light exercise with standard management for adult ED patients with concussion are forthcoming and will also inform ED providers how to counsel patients regarding light exercise. ${ }^{10}$ Thus, based on the best available evidence, current guidelines for the management of concussion recommend no more than 2 days of physical rest and a graded return to activities as tolerated. ${ }^{11}$

As this study by Morrison and colleagues underscores, adults with concussion are frequent patients in the ED, and a minority of these patients will return with persistent symptoms. Although this is a self-limited condition in the majority of patients, there remains an urgent need for a clinical prognostic tool that accurately identifies adult patients at risk for post-concussion syndrome early in their injury. In addition, forthcoming research on the effectiveness of early interventions in acute concussion will likely shed light on ED interventions, which may mitigate the impact of prolonged symptoms in this patient population.

Keywords: Concussion, emergency department, mild traumatic brain injury, post-concussion syndrome

Competing interests: None declared.

\section{REFERENCES}

1. Jagoda AS, Bazarian JJ, Bruns Jr JJ, et al. American College of Emergency Physicians; Centers for Disease Control and Prevention. Clinical policy: neuroimaging and decision making in adult mild traumatic brain injury in the acute setting. Ann Emerg Med 2008;52(6):714-48.

2. Ryan LM, Warden DL. Post-concussion syndrome. Int Rev Psychiatry 2003;15(4):310-6.

3. Morrison L, Taylor R, Mercuri M, Thompson J. Examining Canada's return visits to the emergency department after a concussion. C7EM 2019;21(6):784-788.

4. Stein MB, Jain S, Giacino JT, et al. Risk of posttraumatic stress disorder and major depression in civilian patients after mild traumatic brain injury: a TRACK-TBI Study. 7AMA Psychiatry 2019;76(3):249-58.

5. Shih RA, Schell TL, Hambarsoomian K, Belzberg H, Marshall GN. Prevalence of posttraumatic stress disorder and major depression after trauma center hospitalization. 7 Trauma 2010;69(6):1560-6.

6. Velikonja D, Quon D, Baldisera T, et al. Standards for high quality post-concussion care - post concussion care pathway. Ontario Neurotrauma Foundation; 2018. Available at: http:// concussionsontario.org/standards/tools-resources/post-con cussion-care-pathway/ (accessed August 30, 2019).

7. Eliyahu L, Kirkland S, Campbell S, Rowe BH. The effectiveness of early educational interventions in the emergency department to reduce incidence or severity of post- 
concussion syndrome following a concussion: a systematic review. Acad Emerg Med 2016;23(5):531-42.

8. Grool AM, Aglipay M, Momoli F, et al. Association between early participation in physical activity following acute concussion and persistent postconcussive symptoms in children and adolescents. FAMA 2016;316(23):2504-14.

9. Leddy JJ, Haider MN, Ellis MJ. Early subthreshold aerobic exercise for sport-related concussion. $7 A M A$ Pediatr 2019;173(4):319-25.
10. ClinicalTrials.gov. Bethesda (MD): National Library of Medicine (US). Identifier NCT03084211. Prescribed light exercise for ED patients with MTBI; 2017. Available at: https://clinicaltrials.gov/ct2/show/NCT03084211 (accessed August 30, 2019).

11. Marshall S, Bayley M, McCullagh S, et al. Guideline for Concussion/Mild Traumatic Brain Injury and Persistent Symptoms. 3rd ed. (for Adults 18+ years of age). Toronto: Ontario Neurotrauma Foundation; 2018. 